\title{
Selfcoincidences and roots in Nielsen theory
}

\author{
Ulrich Koschorke
}

\begin{abstract}
Given two maps $f_{1}$ and $f_{2}$ from the sphere $S^{m}$ to an $n$-manifold $N$, when are they loose, i.e. when can they be deformed away from one another? We study the geometry of their (generic) coincidence locus and its Nielsen decomposition. On the one hand the resulting bordism class of coincidence data and the corresponding Nielsen numbers are strong looseness obstructions. On the other hand the values which these invariants may possibly assume turn out to satisfy severe restrictions, e.g. the Nielsen numbers can only take the values 0,1 or the cardinality of the fundamental group of $N$. In order to show this we compare different Nielsen classes in the root case (where $f_{1}$ or $f_{2}$ is constant) and we use the fact that all but possibly one Nielsen class are inessential in the selfcoincidence case (where $f_{1}=f_{2}$ ). Also we deduce strong vanishing results.
\end{abstract}

Mathematics Subject Classification (2000). Primary 54H25, 55M20; Secondary 55N22, 55P35.

Keywords. Coincidence invariants; Nielsen number; looseness obstruction; Hopf invariant.

\section{Introduction}

This work is dedicated to Albrecht Dold whose enormous influence on the development of topology continues to this very day. In fact, it was his stimulating paper [DG] (written jointly with Daciberg Gonçalves) which got me interested in coincidences of maps and which inspired me to introduce a related obstruction theory (cf. [K2 - K7]). Here we apply it to the special case where the domain is a sphere.

Throughout this paper let $f_{1}, f_{2}: S^{m} \rightarrow N$ be two (continuous) maps into an $n$-dimensional smooth connected manifold $N$ without boundary. We will also assume that $m, n \geq 2$ (the remaining cases being well understood).

Supported in part by DAAD (Germany) and CAPES (Brazil). 
We want to make the coincidence locus

$$
\mathrm{C}\left(f_{1}, f_{2}\right)=\left\{x \in S^{m} \mid f_{1}(x)=f_{2}(x)\right\}
$$

as small as possible (in some sense) by deforming $f_{1}$ and $f_{2}$.

Definition 1.2. (compare DG], p. 293-296 and GR2], §1, where a different terminology is used for the same concepts; see also [K7, 5.3).

(i) The pair $\left(f_{1}, f_{2}\right)$ is called loose if there exist maps $f_{i}^{\prime}$ homotopic to $f_{i}$, $i=1,2$, whose coincidence locus $\mathrm{C}\left(f_{1}^{\prime}, f_{2}^{\prime}\right)$ is empty (i.e. " $f_{1}$ and $f_{2}$ can be deformed away from one another").

(ii) In the special case $f_{1}=f_{2}=: f$ we have a refined notion: the pair $(f, f)$ is loose by small deformation if for every metric on $N$ and for every $\varepsilon>0$ there exists an $\varepsilon$-approximation $\bar{f}$ of $f$ such that $\bar{f}(x) \neq f(x)$ for all $x \in S^{m}$.

Proposition 1.3. All pairs of maps $f_{1}, f_{2}: S^{m} \rightarrow N$ are loose if at least one of the following conditions hold:

(i) $m<n$; or

(ii) $N$ is not compact; or

(iii) the fundamental group $\pi_{1}(N)$ is not finite; or

(iv) $N$ is the total space of a Serre fibration with a section and with strictly positive dimensions of the fiber and base (e.g. if $N$ is the product of such spaces).

Proposition 1.4. Assume that

(v) $N$ is noncompact or has trivial Euler characteristic $\chi(N)$ (this holds e.g. when $n$ is odd), or

(vi) $\pi_{m-1}\left(S^{n-1}\right)=0$.

Then for all maps $f: S^{m} \rightarrow N$ the pair $(f, f)$ is loose by small deformation.

Example 1.5 (surfaces). Let $n=2$ and $m \geq 1$. Then all such pairs $(f, f)$ are loose by small deformation except when $m=2$ and $N=S^{2}$ or $\mathbb{R P}(2)$.

It is not hard to verify the looseness claims in 1.3. 1.4 and 1.5 by elementary considerations (see section 2 below). However, in general a deeper analysis is needed and the following approach has proved to be fruitful.

For every pair of (base point preserving) maps $f_{1}, f_{2}: S^{m} \rightarrow N$ we defined (in [K6]) an invariant

$$
\omega^{\#}\left(f_{1}, f_{2}\right) \in \pi_{m}\left(S^{n} \wedge(\Omega N)^{+}\right)
$$

which reflects the geometry of a (generic) coincidence submanifold $C \subset S^{m}$, its normal bundle as well as certain path space data (for details see [K6] or section 3 below). The invariant $\omega^{\#}\left(f_{1}, f_{2}\right)$ depends only on the (base point preserving) homotopy classes of $f_{1}, f_{2}$ and must vanish if $\left(f_{1}, f_{2}\right)$ is loose (and, in particular, in all cases listed in proposition 1.3. . The converse holds in a "stable" dimension range.

Theorem 1.7. (compare $\mathrm{K} 4,1.10$ and $\mathrm{K} 2$, 2.2). Assume $m<2 n-2$. Then a pair $\left(f_{1}, f_{2}\right)$ is loose precisely if $\omega^{\#}\left(f_{1}, f_{2}\right)=0$. In the special case $f_{1}=f_{2}=: f$ the pair $(f, f)$ is loose by small deformation precisely if $\omega^{\#}(f, f)=0$. 
Our looseness obstruction is always compatible with additions in homotopy groups:

$$
\omega^{\#}\left(f_{1}+f_{1}^{\prime}, f_{2}+f_{2}^{\prime}\right)=\omega^{\#}\left(f_{1}, f_{2}\right)+\omega^{\#}\left(f_{1}^{\prime}, f_{2}^{\prime}\right)
$$

for all $\left[f_{i}\right],\left[f_{i}^{\prime}\right] \in \pi_{m}(N), i=1,2$.

There is also a canonical involution inv on the group $\pi_{m}\left(S^{n} \wedge(\Omega N)^{+}\right)$. It plays a rôle e.g. in the symmetry relation

$$
\omega^{\#}\left(f_{2}, f_{1}\right)=\operatorname{inv}\left(\omega^{\#}\left(f_{1}, f_{2}\right)\right) .
$$

Two special settings are very central in coincidence theory.

I. The root case: $f_{2}=y_{0}$ (where the fixed value $y_{0} \in N$ is given).

Here our invariant yields the degree homomorphism

$$
\operatorname{deg}^{\#}:=\omega^{\#}\left(-, y_{0}\right): \pi_{m}(N) \rightarrow \pi_{m}\left(S^{n} \wedge(\Omega N)^{+}\right) .
$$

(For a purely homotopy theoretical interpretation in terms of an enriched HopfGanea invariant see theorem 7.2 in $\mathrm{K} 6$.)

Clearly deg ${ }^{\#}$ vanishes on $i_{*}\left(\pi_{m}(N \backslash\{*\})\right)$ where $i: N \backslash\{*\} \hookrightarrow N$ denotes the inclusion of the complement of a point $*$ in $N$. It turns out that the resulting sequence

$$
\pi_{m}(N \backslash\{*\}) \stackrel{i_{*}}{\longrightarrow} \pi_{m}(N) \stackrel{\text { deg }^{\#}}{\longrightarrow} \pi_{m}\left(S^{n} \wedge(\Omega N)^{+}\right)
$$

is very often exact, e.g. when $m \leq 2 n-3$ or $n \leq 2$ or $N$ is a sphere or a (real, complex or quaternionic) projective space of arbitrary dimension (cf. [K6], (6.5)). Thus in these cases $\operatorname{deg}^{\#}(f)$ is the complete looseness obstruction for the pair $\left(f, y_{0}\right)$.

Question 1.12. Is the sequence (1.11) always exact?

The degree deg $^{\#}$ can be considered to be our basic coincidence invariant since (in view of (1.8) and (1.9))

$$
\begin{aligned}
\omega^{\#}\left(f_{1}, f_{2}\right) & =\omega^{\#}\left(f_{1}, y_{0}\right)+\omega^{\#}\left(y_{0}, f_{2}\right) \\
& =\operatorname{deg}^{\#}\left(f_{1}\right)+\operatorname{inv}\left(\operatorname{deg}^{\#}\left(f_{2}\right)\right)
\end{aligned}
$$

for all $\left[f_{1}\right],\left[f_{2}\right] \in \pi_{m}\left(N, y_{0}\right)$.

II. The selfcoincidence case: $f_{1}=f_{2}=: f$.

Consider the bundle $S T(N)$ of unit tangent vectors over $N$ (with respect to some metric) and the resulting exact (horizontal) homotopy sequence as well as the Freudenthal suspension homomorphism $E$ :

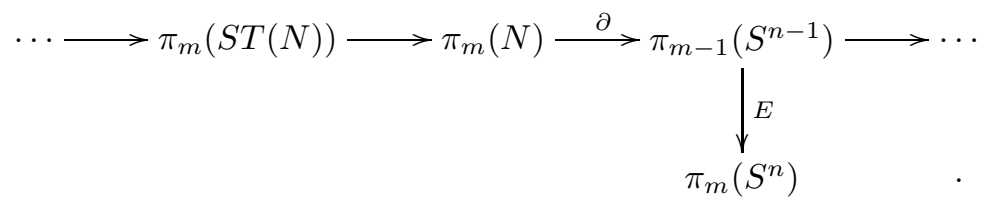


Theorem 1.15. Given $[f] \in \pi_{m}(N)$, we have the following logical implications:

(i) $\partial([f]) \in \pi_{m-1}\left(S^{n-1}\right)$ vanishes;

\

(ii) $(f, f)$ is loose by small deformation;

$\Downarrow \quad(\Uparrow$ if $N=\mathbb{R} \mathbb{P}(n))$

(iii) $(f, f)$ is loose (by any deformation);

$\Downarrow \quad\left(\Uparrow\right.$ if $\left.N=S^{n}\right)$

(iv) $\omega^{\#}(f, f)=0$;

立

(v) $E(\partial([f])=0$.

Thus $\omega^{\#}(f, f)$ is just "one desuspension short" of being the complete looseness obstruction.

The equivalence of (i) and (ii) was already noted by Dold and Gonçalves in DG.

Observe also that all conditions (i)-(v) in [1.15] except (iii) are compatible with covering projections $p: \tilde{N} \rightarrow N$ (compare [K7,1.22).

Corollary 1.16. The conditions (i),..,(v) in 1.15 are all equivalent if the suspension homomorphism $E$, when restricted to $\partial\left(\pi_{m}(N)\right)$ (cf. (1.14)), is injective and, in particular, if $m \leq n+3$ or if $m=n+4 \neq 10$ or in the stable dimensional range $m \leq 2 n-3$.

Indeed, in these three dimension settings $E$ is injective whenever $n \equiv 0(2)$. Clearly conditions (i)-(v) in 1.15]are automatically satisfied under the assumptions of proposition 1.4

Example 1.17 $\left(N=\mathbb{R} \mathbb{P}(n)\right.$ or $\left.S^{n}\right)$. If $m \leq n+4$, then the five conditions in 1.15)are equivalent for all maps $f: S^{m} \rightarrow \mathbb{R} \mathbb{P}(n)$ and $\tilde{f}: S^{m} \rightarrow S^{n}$ (even in the exceptional case $m=n+4=10$ since $\left.\pi_{10}\left(S^{6}\right)=0\right)$.

However, this is no longer true for $m=n+5=11$. Indeed, according to [T] and $\mathrm{P}$ we have in (1.14)

$$
\frac{1}{2} H: \pi_{11}\left(S^{6}\right) \stackrel{\cong}{\longrightarrow} \mathbb{Z} ; \quad \pi_{10}\left(S^{5}\right) \cong \mathbb{Z}_{2} ; \quad \pi_{10}\left(V_{7,2}\right)=0
$$

where $H$ denotes the Hopf invariant. Thus $\partial$ is onto, but $E$ and hence $E \circ \partial$ is trivial here. Therefore, given any map $f: S^{11} \rightarrow \mathbb{R P}(6)$ and a lifting $\tilde{f}: S^{11} \rightarrow S^{6}$ of it, the invariants $\omega^{\#}(f, f)$ and $\omega^{\#}(\tilde{f}, \tilde{f})$ vanish and the pair $(\tilde{f}, \tilde{f})$ is loose (see [K6], 1.12; compare also [GW]). But $(f, f)$ is loose and, equivalently, $(\tilde{f}, \tilde{f})$ is loose by small deformation only (and precisely) if $[f] \in 2 \cdot \pi_{11}(\mathbb{R P}(6)$ ) or, equivalently, if $H(\tilde{f}) \equiv 0(4)$ (compare [GR1] and [K7, 1.19; the delicate difference between conditions (ii) and (iii) in 1.15 is further illustrated e.g. in [GR2] and [K7, 1.22). 
For simple examples of nontrivial $\omega^{\#}$-values consider the case $m=n$. If a map $\tilde{f}: S^{n} \rightarrow S^{n}$ has (standard) mapping degree $d$, then

$$
\pm \omega^{\#}(\tilde{f}, \tilde{f})=E \circ \partial(\tilde{f})=\left(1+(-1)^{n}\right) d
$$

in $\pi_{n}\left(S^{n} \wedge\left(\Omega S^{n}\right)^{+}\right) \cong \pi_{n}\left(S^{n}\right) \cong \mathbb{Z}$. Assume that $n$ is even. Then $(\tilde{f}, \tilde{f})$ is loose if and only if $\tilde{f}$ is nullhomotopic; the same holds for maps from $S^{n}$ into $\mathbb{R} \mathbb{P}(n)$. This shows that the exception made in example 1.5 is indeed necessary.

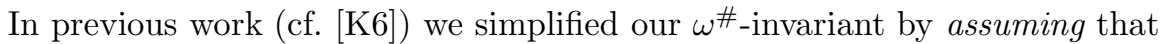
$f_{1}$ or $f_{2}$, say $f_{2}$, is "not coincidence producing" (cf. BS]), i.e.

$$
\left(\bar{f}_{2}, f_{2}\right) \text { is loose for some map } \bar{f}_{2}: S^{m} \rightarrow N \text {. }
$$

Then $\left(f_{1}, f_{2}\right)$ and $\left(f_{1}-\bar{f}_{2},\left(f_{2}-f_{2}\right) \sim y_{0}\right)$ have a similar coincidence behaviour and

$$
\omega^{\#}\left(f_{1}, f_{2}\right)=\operatorname{deg}^{\#}\left(f_{1}-\bar{f}_{2}\right) .
$$

In this paper we start from the decomposition (up to homotopy)

$$
\left(f_{1}, f_{2}\right) \sim\left(f_{1}-f_{2}, y_{0}\right)+\left(f_{2}, f_{2}\right)
$$

which is always available without any assumption. In view of (1.8) this implies the basic equation

$$
\omega^{\#}\left(f_{1}, f_{2}\right)=\operatorname{deg}^{\#}\left(f_{1}-f_{2}\right)+\omega^{\#}\left(f_{2}, f_{2}\right) .
$$

Vanishing results concerning the second ("selfcoincidence") summand are not only interesting in view of theorem 1.15, but they also allow us to reduce our analysis to studying the degree homomorphism deg $\#$ or, equivalently, Hopf-Ganea invariants (cf. [K6], 7.2).

It turns out that the cardinality $\# \pi_{1}(N) \in \mathbb{Z} \cup\{\infty\}$ of the fundamental group of $N$ plays a crucial rôle.

Theorem 1.21. Assume that

(i) $\# \pi_{1}(N)>2$ and $N$ is orientable or not; or

(ii) $\# \pi_{1}(N) \geq 2$ and $N$ is orientable.

Then $\omega^{\#}(f, \bar{f})=0$ for all $f: S^{m} \rightarrow N$.

In particular

$$
\omega^{\#}\left(f_{1}, f_{2}\right)=\operatorname{deg}^{\#}\left(f_{1}-f_{2}\right)
$$

for all $\left[f_{1}\right],\left[f_{2}\right] \in \pi_{m}(N)$. relation

Also the set of all possible values of our $\omega^{\#}$-invariant is restricted by the

$$
\left\{\omega^{\#}\left(f_{1}, f_{2}\right)\right\}=\operatorname{im}(\operatorname{deg} \#) \subset \operatorname{ker}(\mathrm{id}+\mathrm{inv})
$$

where $\mathrm{id}=$ identity and inv denotes the canonical involution of $\pi_{m}\left(S^{n} \wedge(\Omega N)^{+}\right)$ (cf. (1.9) and (1.13)).

If in addition $m<2 n-2$ or $m \leq n+3$, then the pair $(f, f)$ is loose by small deformation for every map $f: S^{m} \rightarrow N$. 
On the one hand, the proof (given in section 4 below) compares the contributions of the pathcomponents of the loop space $\Omega N$ to $\omega^{\#}(f, f)$; on the other hand, it uses the fact that at most one such pathcomponent can contribute nontrivially to our selfcoincidence invariant.

Example 1.22. As an application consider the case where $m=n$ and $N$ is an orientable $n$-manifold with $\pi_{1}(N)=\mathbb{Z}_{2}$. Then

$$
\text { inv }=(-1)^{n} \text { id } \quad \text { on } \quad \pi_{n}\left(S^{n} \wedge(\Omega N)^{+}\right) \cong \mathbb{Z} \oplus \mathbb{Z} .
$$

If $n$ is even then $\operatorname{ker}(\mathrm{id}+\mathrm{inv})=0$; therefore each pair of maps $f_{1}, f_{2}: S^{m} \rightarrow N$ is loose (since $\omega^{\#}\left(f_{1}, f_{2}\right)=0$ ).

Example 1.23 (real Grassmann manifolds). Consider the manifold $G_{r, k}$ (or $\tilde{G}_{r, k}$, resp.) of nonoriented (or oriented, resp.) $k$-planes in $\mathbb{R}^{r}, 0<k<r$. If $r$ is even, then $G_{r, k}$ is orientable, $\pi_{1}\left(G_{r, k}\right) \neq\{0\}$ and hence $\omega^{\#}(f, f)=0$ for all maps from $S^{m}, m \geq 1$, to $G_{r, k}$ or $\tilde{G}_{r, k}$. This does not seem to follow from 1.3 or 1.4 indeed, the Euler characteristic of $G_{r, k}$ is strictly positive if both $r$ and $k$ are even (see 4.3 below; compare also our example 2.2).

A further vanishing result (quoted from [K6],1.9; compare also [K7], 5.4) is of interest here.

Proposition 1.24. Assume $\pi_{1}(N) \neq 0$. Given $[f] \in \pi_{m}(N), m \geq 2$, if $j_{*} \circ \partial([f])$ vanishes then so does $\omega^{\#}(f, f)$.

Here $j: S^{n-1} \hookrightarrow N \backslash\{*\}$ denotes the inclusion of the boundary sphere of a small $n$-ball in $N$ centered at a point $* \in N$. According to [K7, 5.4, the condition $j_{*} \circ \partial([f])=0$ just means that $f$ is not coincidence producing (cf. (1.18)).

Let us summarize: although our selfcoincidence invariant $\omega^{\#}(f, f)$ is "only one desuspension short" of being a complete looseness obstruction, it vanishes for a great number of maps $f$ defined on spheres (in contrast, in [K2 $\omega^{\#}(f, f)$ was shown to be highly nontrivial in many examples where the domain of $f$ is a general closed manifold $M$ ).

Corollary 1.25. Assume $\pi_{1}(N) \neq 0$ and $n \geq 1$. If $\omega^{\#}(f, f) \neq 0$ for a map $f: S^{m} \rightarrow$ $N$ then the following restrictions must all be satisfied:

a) $n$ is even and $m \geq n \geq 4$, or else $m=2$ and $N=\mathbb{R P}(2)$; and

b) $N$ is closed and nonorientable, $\pi_{1}(N)=\mathbb{Z}_{2}, \quad \chi(N) \neq 0$; moreover the homomorphism $i_{*}: \pi_{m}\left(N \backslash\{*\}, y_{0}\right) \rightarrow \pi_{m}\left(N, y_{0}\right)$ (induced by the inclusion of $N$, punctured at some point $* \neq y_{0}$ ) is not onto; furthermore, $N$ does not allow a free smooth action by a nontrivial finite group; and

c) $E \circ \partial([f]) \neq 0$ and $j_{*} \circ \partial([f]) \neq 0$ (compare (1.14) and 1.21).

Thus an obvious place to look for nontrivial values of $\omega^{\#}(f, f)$ are evendimensional real projective spaces (cf. already example 1.17). 
Example 1.26. If $n=4,8,12,14,16$ or 20 , then there exist infinitely many homotopy classes $[f] \in \pi_{2 n-1}(\mathbb{R} \mathbb{P}(n))$ such that $\omega^{\#}(f, f) \neq 0$.

We see this with the help of the weaker invariant $\omega(f, f) \in \pi_{m-n}^{S}$ (cf. (3.15) below and [K2, 2.1), which stabilizes and simplifies $\omega^{\#}(f, f)$ and is more easily computable.

However, for many maps $f, f_{1}, f_{2}$ from $S^{m}$ into a nonorientable $n$-manifold $N$ this approach does not yield nontrivial $\omega$-values. Indeed, $\omega(f, f)=0$ whenever $2 \cdot \pi_{m-n}^{S}=0$, e.g. when $m-n=1,2,4,5,6,8,9,12,14,16$ or 17 (cf. [T]). Moreover, for all $m$ and $n$

$$
(\chi(N)-1) \omega\left(f_{1}, f_{2}\right)=0
$$

where $\chi(N)$ stands for the Euler characteristic of $N$.

Example $1.28\left(\mathbf{N}=\mathbf{G}_{\mathbf{5}, \mathbf{2}}\right.$; compare 1.23) . The invariant $\omega\left(f_{1}, f_{2}\right)$ vanishes for all maps $f_{1}, f_{2}: S^{m} \rightarrow G_{5,2}, m \geq 1$. In particular, the induced homomorphism

$$
\operatorname{coll}_{*}: \pi_{m}\left(G_{5,2}\right) \rightarrow \pi_{m}\left(S^{6}\right)
$$

is trivial for $m \leq 10$ where coll denotes the degree one map which collapses all but an open topdimensional ball into a point. (Note that e.g. for $m=6,7$ or 8 , resp., $\pi_{m}\left(G_{5,2}\right) \cong \pi_{m}\left(V_{5,2}\right)$ is isomorphic to $\mathbb{Z}_{2}, \mathbb{Z} \oplus \mathbb{Z}_{2}$ and $\mathbb{Z}_{2}$, resp.; cf. $[\mathrm{P}$ ).

In section 4 below we will deduce the vanishing theorem 1.21 and relations such as (1.27) and 1.28 from a careful analysis of the root case in section 3

While our looseness obstructions lie in complicated groups which are usually hard to compute, they give rise to simple numerical invariants (defined in section 5 below). These generalize the Nielsen numbers which have played such a central rôle in topological fixed point theory (cf. e.g. [B] and [K6, 1.12 (iv)). In analogy to the classical procedure, our Nielsen numbers $\mathrm{N} \#\left(f_{1}, f_{2}\right) \geq \mathrm{N}\left(f_{1}, f_{2}\right)$ count those "Reidemeister classes" $A \in \pi_{0}(\Omega N)=\pi_{1}(N)$ which make an essential contribution to $\omega^{\#}\left(f_{1}, f_{2}\right)$ and $\tilde{\omega}\left(f_{1}, f_{2}\right)$, resp.

Theorem 1.29. (cf. [K6], 1.2). For every pair $\left(f_{1}^{\prime}, f_{2}^{\prime}\right)$ of maps homotopic to $\left(f_{1}, f_{2}\right)$ the number of pathcomponents of the coincidence subspace $\mathrm{C}\left(f_{1}^{\prime}, f_{2}^{\prime}\right) \subset S^{m}$ is at least $\mathrm{N}^{\#}\left(f_{1}, f_{2}\right)$.

There are quite a few examples where $\mathrm{N}^{\#}\left(f_{1}, f_{2}\right)$ is the best possible such lower bound (see e.g. the "Wecken theorems" in [K6], 1.3, 1.12, 1.13, 1.14,...).

Theorem 1.30. For all maps $f_{1}, f_{2}: S^{m} \rightarrow N$ we have:

$$
\begin{gathered}
\mathrm{N} \#\left(f_{1}, f_{2}\right)=0 \quad \text { if and only if } \quad \omega^{\#}\left(f_{1}, f_{2}\right)=0 ; \\
\mathrm{N}\left(f_{1}, f_{2}\right)=0 \quad \text { if and only if } \quad \tilde{\omega}\left(f_{1}, f_{2}\right)=0 .
\end{gathered}
$$

We call this the norm property of our Nielsen numbers: they decide whether elements in the image group of our $\omega$-invariants are zero, just like norms of vectors do. (Recall, in addition, analogues of the triangular inequality, cf. [K6], 6.2.)

By definition $\mathrm{N}^{\#}\left(f_{1}, f_{2}\right)$ is among the integers between 0 and $\# \pi_{1}(N)$. But few of these actually occur as Nielsen numbers. 
Theorem 1.31. Let $k \in \mathbb{Z} \cup\{\infty\}$ be the number of elements in $\pi_{1}(N)$. Then for each pair of maps $f_{1}, f_{2}: S^{m} \rightarrow N$ the Nielsen numbers $\mathrm{N}^{\#}\left(f_{1}, f_{2}\right)$ and $\mathrm{N}\left(f_{1}, f_{2}\right)$ may assume only the two values 0 or $k$ or, if $k=2$ and $N$ is an even-dimensional, closed, nonorientable manifold with nontrivial Euler characteristic, also 1 as a third possible value.

Here again (as in [K7]) the case $N=\mathbb{R} \mathbb{P}(n)$ turns out to be particularly interesting. E.g. assume that $m=n$ is even and let $p: S^{n} \rightarrow \mathbb{R P}(n)$ be the canonical covering map. Then $\mathrm{N}^{\#}\left(f_{1}, f_{2}\right)=\mathrm{N}\left(f_{1}, f_{2}\right)$ equals 0,1 and 2, resp., when $\left(f_{1}, f_{2}\right)=\left(y_{0}, y_{0}\right),(p, p)$ and $\left(p, y_{0}\right)$, resp. (cf. the end of section 4 below).

Remark 1.32. It will be convenient to consider base point preserving maps and homotopies in sections 3 5 below. Recall, however, that looseness and Nielsen numbers depend only on free homotopy classes and so does the vanishing of our $\omega$-invariants (see e.g. [K6], 1.2, 2.1 and the appendix there).

Remark 1.33. Our approach can also be extended to the setting of fibre preserving maps between smooth fibrations. The resulting coincidence invariants are closely related to A. Dold's fixed point index which was defined and studied in D].

Conventions and Notations. Throughout this paper $N$ denotes a smooth connected manifold without boundary (Hausdorff and having a countable basis). Our notation will often not distinguish between a constant map and its value.

\section{Acknowledgment}

It is a pleasure to thank D. Gonçalves and E. Kudryavtseva for stimulating discussions.

\section{Looseness}

In this section we use rather elementary techniques to establish the looseness results in propositions 1.3 and 1.4 as well as example 1.5 .

Lemma 2.1. Let $y_{1} \neq y_{2}$ be different points in $N$ and assume that the homomorphism $i_{*}: \pi_{m}\left(N \backslash\left\{y_{1}\right\}, y_{2}\right) \rightarrow \pi_{m}\left(N, y_{2}\right)$ induced by the inclusion map is onto. Then for all maps $f_{1}, f_{2}: S^{m} \rightarrow N$ the pair $\left(f_{1}, f_{2}\right)$ is loose.

Proof. Let $S_{+}^{m}$ and $S_{-}^{m}$ denote the hemispheres defined by $x_{1} \geq 0$ and $x_{1} \leq 0$, resp., $x=\left(x_{1}, \ldots, x_{m+1}\right) \in S^{m}$. Then $f_{2}$ is homotopic to a map $f_{2}^{\prime}$ such that $f_{2}^{\prime}\left(S_{+}^{m}\right) \subset N \backslash\left\{y_{1}\right\}$ and $\left.f_{2}^{\prime}\right|_{S_{-}^{m}} \equiv y_{2}$. Similarly, $f_{1} \sim f_{1}^{\prime}$ such that $\left.f_{1}^{\prime}\right|_{S_{+}^{m}} \equiv y_{1}$ and $f_{1}^{\prime}\left(S_{-}^{m}\right) \subset N \backslash\left\{y_{2}\right\}$. Clearly the pair $\left(f_{1}^{\prime}, f_{2}^{\prime}\right)$ is coincidence free.

Proposition 1.3 follows as a corollary since each of the conditions (i) through (iv) imply that $i_{*}$ is onto. If $m<n$, this is seen by making a map $f: S^{m} \rightarrow N$ transverse to $\left\{y_{1}\right\}$. If $N$ is not compact, deform $f$ by an isotopy along a smoothly embedded path which starts in $N \backslash f\left(S^{m}\right)$, ends in $y_{1}$ and avoids $y_{2}$.

If the universal covering space $p: \tilde{N} \rightarrow N$ has infinitely many layers, consider a lifting $\tilde{f}: S^{m} \rightarrow \tilde{N}$ of $f$. Apply an isotopy along suitable disjoint paths in $\tilde{N}$ 
which start in $\tilde{N} \backslash \tilde{f}\left(S^{m}\right)$ and end in the finitely many points of $\tilde{f}\left(S^{m}\right) \cap p^{-1}\left(\left\{y_{1}\right\}\right)$. The corresponding homotopy in $N$ deforms $f$ into $N \backslash\left\{y_{1}\right\}$.

Finally, in case (iv) of proposition 1.3 the exact homotopy sequence of the fibration splits. Thus $f$ can be deformed into the union of the fibre and the image of the section.

Example 2.2. For $r=2 r^{\prime}>2$ and $m \geq 1$ every pair of maps $f_{1}, f_{2}: S^{m} \rightarrow G_{r, 2}$ (into the Grassmann manifold of 2-planes in $\mathbb{R}^{r}$ ) is loose.

Indeed, due to the complex structure on $\mathbb{R}^{r}=\mathbb{C}^{r^{\prime}}$, the fibration $S^{r-2} \hookrightarrow$ $V_{r, 2} \rightarrow S^{r-1}$ (of the Stiefel manifold of 2-frames in $\mathbb{R}^{r}$ ) has a canonical section; hence

$$
\pi_{m}\left(V_{r, 2}\right) \cong \pi_{m}\left(S^{r-2}\right) \oplus \pi_{m}\left(S^{r-1}\right) .
$$

For $m \geq 3$ this group is also isomorphic to $\pi_{m}\left(G_{r, 2}\right)$, and the summands correspond to the subspaces

$$
A:=\left\{2 \text {-planes containing the base vector } e_{r}\right\}
$$

and

$$
B:=\left\{\text { complex lines in } \mathbb{C}^{r^{\prime}}\right\} .
$$

Since $A \cup B \varsubsetneqq G_{r, 2}$, our claim follows from lemma 2.1.

Proof of proposition 1.4. A homotopy lifting argument shows that $(f, f)$ is loose by small deformation if and only if the pulled back bundle $f^{*}(T N)$ has a nowhere vanishing section over $S^{m}$ (compare [DG]). If $N$ is noncompact or $\chi(N)=0$, then the tangent bundle $T N$ itself has a nonzero section over $f\left(S^{m}\right)$ which we can pull back. In any case every $n$-plane bundle over $S^{m}$ allows a section with a single zero; it can be removed if its "index map" $q: S^{m-1} \rightarrow S^{n-1}$ (compare Wy or also [K6], (28)) is nullhomotopic.

The claim in example 1.5 follows since $\pi_{m-1}\left(S^{1}\right)=0$ except when $m=2$. Furthermore $\pi_{2}(N)=0$ for every surface other than $N=S^{2}$ or $\mathbb{R P}(2)$.

\section{Looseness obstructions}

In this section we recall the definitions of the various versions of the $\omega$-invariants. (For more details we refer to [K6; see also K4 and [K2.) Moreover we compare the Nielsen components of $\omega^{\#}$ in the root case (in [3.12). This will be needed to establish our main results.

Unless specified otherwise we will assume that $m, n \geq 2$. Fix basepoints $x_{0} \in S^{m}$ and $y_{0} \in N$, and a local orientation of $N$ at $y_{0}$. Throughout the remainder of this paper we consider (continuous) maps $f_{1}, f_{2}, f, \ldots:\left(S^{m}, x_{0}\right) \rightarrow\left(N, y_{0}\right)$. Our notation will not distinguish between a constant map and its value.

After a suitable approximation we may assume that $\left(f_{1}, f_{2}\right): S^{m} \rightarrow N \times N$ is smooth and transverse to the diagonal

$$
\Delta=\{(y, y) \in N \times N \mid y \in N\} .
$$


Then the coincidence set

$$
C=\mathrm{C}\left(f_{1}, f_{2}\right)=\left(f_{1}, f_{2}\right)^{-1}(\Delta)=\left\{x \in S^{m} \mid f_{1}(x)=f_{2}(x)\right\}
$$

is a smooth closed $n$-codimensional submanifold of $S^{m}$. The map $\left(f_{1}, f_{2}\right)$ induces an isomorphism of (normal) vector bundles

$$
\left.\nu\left(C, S^{m}\right) \cong\left(f_{1}, f_{2}\right)^{*}(\nu(\Delta, N \times N)) \cong f_{1}^{*}(T N)\right|_{C} .
$$

Now pick a homotopy $G: C \times I \rightarrow S^{m}$ which deforms the inclusion map $g: C \hookrightarrow$ $S^{m}$ to the constant map with value $x_{0}$. (Such a contraction exists and is unique up to homotopy rel $(0,1)$ since $n \geq 2$ and the complement of any point in $S^{m}$ allows "linear" homotopies.) Then $G$ induces a vector bundle isomorphism from $\left.f_{1}^{*}(T N)\right|_{C}=g^{*}\left(f_{1}^{*}(T N)\right)$ to $C \times T_{y_{0}}(N)$. Composing this with (3.2) and using our choice of a local orientation of $N$ at $y_{0}$, we get a framing

$$
\bar{g}^{\#}: \nu\left(C, S^{m}\right) \stackrel{\cong}{\longrightarrow} C \times \mathbb{R}^{n} .
$$

Furthermore we obtain the map

$$
\tilde{g}: C \rightarrow \Omega\left(N, y_{0}\right)=: \Omega N
$$

which assigns the (concatenated) loop

$$
y_{0}=f_{1}\left(x_{0}\right) \stackrel{\left(f_{1} \circ G(x,-)\right)^{-1}}{\longrightarrow} f_{1}(x)=f_{2}(x) \stackrel{f_{2} \circ G(x,-)}{\longrightarrow} f_{2}\left(x_{0}\right)=y_{0}
$$

to $x \in C$.

The resulting bordism class

$$
\omega^{\#}\left(f_{1}, f_{2}\right)=\left[C, \bar{g}^{\#}, \tilde{g}\right]
$$

of the framed compact submanifold $C \subset S^{m}$ (cf. (3.1); (3.3)) together with the map $\tilde{g}$ (cf. (3.4) $)$ depends only on the homotopy classes $\left[f_{i}\right] \in \pi_{m}\left(N, y_{0}\right), i=1,2$. Via the Pontryagin-Thom procedure, $\omega^{\#}\left(f_{1}, f_{2}\right)$ can also be interpreted as an element in the $m$-th homotopy group of the Thom space $S^{n} \wedge(\Omega N)^{+}$of the trivial $n$-plane bundle over the loop space $\Omega N$. (Here "+" stands for a disjointly added point. Note that the bordism theories of submanifolds in $S^{n}$ and $\mathbb{R}^{n}$ are equivalent in codimensions $n \geq 2$; thus it is not necessary that $f_{1}\left(x_{0}\right) \neq f_{2}\left(x_{0}\right)$, as was assumed in [K6].)

If we ignore the map $\tilde{g}$ we obtain the simpler invariant

$$
\underline{\omega}^{\#}\left(f_{1}, f_{2}\right)=\left[C, \bar{g}^{\#}\right] \in \pi_{m}\left(S^{n}\right) .
$$

However, often this means a considerable loss of information. Indeed, in general the loop space $\Omega N$ has a very rich topology and, in particular, can be highly disconnected. Already its decomposition into pathcomponents leads to the important "Nielsen decomposition" of coincidence sets, as follows.

Given a pathcomponent $A \in \pi_{0}(\Omega N)=\pi_{1}\left(N, y_{0}\right)$, restrict your attention to the corresponding partial coincidence manifold

$$
C_{A}:=\mathrm{C}_{A}\left(f_{1}, f_{2}\right):=\tilde{g}^{-1}(A) \quad \subset \mathrm{C}\left(f_{1}, f_{2}\right)
$$


which is again a closed $n$-codimensional submanifold of $S^{n}$ and endowed with the restricted framing $\bar{g}_{A}^{\#}=\bar{g}^{\#} \mid$ and the map $\tilde{g}_{A}=\tilde{g} \mid: C_{A} \rightarrow A \subset \Omega N$. This leads to the invariants

$$
\omega_{A}^{\#}\left(f_{1}, f_{2}\right)=\left[C_{A}, \bar{g}_{A}^{\#}, \tilde{g}_{A}\right] \in \pi_{m}\left(S^{n} \wedge A^{+}\right)
$$

and

$$
\underline{\omega}_{A}^{\#}\left(f_{1}, f_{2}\right)=\left[C_{A}, \bar{g}_{A}^{\#}\right] \in \pi_{m}\left(S^{n}\right) .
$$

Example 3.10. For all $[f] \in \pi_{m}\left(N, y_{0}\right)$ we have $\underline{\omega}^{\#}\left(y_{0}, f\right)=\operatorname{coll}_{*}([f])$. Here

$$
\text { coll }: N \rightarrow N /\left(N \backslash \stackrel{\circ}{B^{n}}\right)=B^{n} / \partial B^{n}=S^{n}
$$

is the map which collapses the complement of a small ball $B^{n}$ near $y_{0} \in \partial B^{n}$ to a point and preserves the local orientation of $N$.

In the root case we will now compare the various Nielsen components of $\omega^{\#}$.

Note that there are two canonical isomorphisms

$$
\varrho_{A *}, \lambda_{A *}: \pi_{m}\left(S^{n} \wedge A_{0}^{+}\right) \stackrel{\cong}{\longrightarrow} \pi_{m}\left(S^{n} \wedge A^{+}\right)
$$

(compare (3.8) ) where $A_{0}$ denotes the pathcomponent of $\Omega N$ containing the constant loop. They are induced by the homotopy equivalences $\varrho_{A}, \lambda_{A}: A_{0} \rightarrow A$ which compose each loop $\ell \in A_{0}$ to the right (or left, resp.) with a fixed loop $\ell_{A} \in A$; e.g. $\varrho_{A}(\ell)$ travels first along $\ell$ and then along $\ell_{A}$.

To simplify notation we will write $\omega_{0}^{\#}$ for $\omega_{A_{0}}^{\#}$.

Proposition 3.12. Given a map $f:\left(S^{m}, x_{0}\right) \rightarrow\left(N, y_{0}\right)$ and $A \in \pi_{1}(N)$, we have

$$
\omega_{A}^{\#}\left(f, y_{0}\right)=\varrho_{A *}\left(\omega_{0}^{\#}\left(f, y_{0}\right)\right) \quad \text { and } \quad \underline{\omega}_{A}^{\#}\left(f, y_{0}\right)=\underline{\omega}_{0}^{\#}\left(f, y_{0}\right)
$$

as well as

$$
\omega_{A}^{\#}\left(y_{0}, f\right)=\iota_{A *} \circ \lambda_{A *}\left(\omega_{0}^{\#}\left(f, y_{0}\right)\right) \quad \text { and } \quad \underline{\omega}_{A}^{\#}\left(y_{0}, f\right)=\iota_{A *}\left(\underline{\omega}_{0}^{\#}\left(y_{0}, f\right)\right)
$$

where $\iota_{A *}$ composes the framing with an orientation preserving (or reversing) automorphism of $\mathbb{R}^{n}$ according as $A$ is (or is not) orientation preserving, i.e. the tangent bundle of $N$, when pulled by $\ell_{A}: S^{1} \rightarrow N,\left[\ell_{A}\right]=A$, becomes trivial or, equivalently, A lies in the kernel of the composed homomorphism

$$
w_{1}(N): \pi_{1}\left(N, y_{0}\right) \rightarrow H_{1}(N) \rightarrow \mathbb{Z}_{2}
$$

which evaluates the first Stiefel-Whitney class of $N$.

Proof (for some of the following arguments compare also the proof of theorem 4.3 in [K6]).

In view of proposition 1.3 (iii) we need to consider only the case when $\pi_{1}(N)$ is finite. Thus the fibre $p^{-1}\left(\left\{y_{0}\right\}\right)$ in the universal covering space $p: \tilde{N} \rightarrow N$ consists of points $\left\{\tilde{y}_{1}, \ldots, \tilde{y}_{k}\right\}$ which all lie in a suitable embedded $n$-ball $V$ in $\tilde{N}$. Now lift $f$ to a map $\tilde{f}: S^{m} \rightarrow \tilde{N}$. After a suitable homotopy we may assume 
that $\tilde{f}$ is smooth, with regular value $\tilde{y}_{1}$, and agrees on a tubular neighbourhood $U \cong \tilde{C} \times V \subset S^{m}$ of $\tilde{C}:=\tilde{f}^{-1}\left(\left\{\tilde{y}_{1}\right\}\right)$ with the projection to $V \subset \tilde{N}$. Then

$$
\mathrm{C}\left(f, y_{0}\right)=\mathrm{C}\left(y_{0}, f\right)=f^{-1}\left(\left\{y_{0}\right\}\right)=\tilde{f}^{-1}\left(\left\{\tilde{y}_{1}, \ldots, \tilde{y}_{k}\right\}\right)
$$

consists of the "parallel" copies

$$
\tilde{C}_{i}:=\tilde{C} \times\left\{\tilde{y}_{i}\right\} \subset U, \quad i=1, \ldots, k .
$$

Note that the straight path $\gamma_{i j}$ joining $\tilde{y}_{i}$ to $\tilde{y}_{j}$ in the ball $V$ yields an isotopy which moves $\tilde{C}_{i}$ to $\tilde{C}_{j}$ in $U \subset S^{m}, 1 \leq i, j \leq k$. We can compose it with a contraction $\left.G\right|_{\tilde{C}_{j} \times I}$ of $\tilde{C}_{j}$ in order to get the required contraction of $\tilde{C}_{i}$ in $S^{m}$. This extra part of the contraction induces a concatenation of the path in (3.5) with the loop $p \circ \gamma_{i j}^{\mp 1}$. On the other hand the isotopy is compatible with the framings of $\tilde{C}_{i}$ and $\tilde{C}_{j}$ if $p \circ \gamma_{i j}$ preserves the local orientation of $N$ or in case we are dealing with $\omega^{\#}\left(f, y_{0}\right)$ since then

$$
\nu\left(\tilde{C}_{t}, S^{n}\right) \cong \tilde{C}_{t} \times\left. T V \cong f^{*}(T N)\right|_{\tilde{C}_{t}},
$$

$0 \leq t \leq 1$, throughout the isotopy. Thus let us consider $\omega^{\#}\left(y_{0}, f\right)$. Here $\tilde{C}_{i}$ and $\tilde{C}_{j}$ are framed via the orientations of $T_{\tilde{y}_{i}} \tilde{N}$ and $T_{\tilde{y}_{j}} \tilde{N}$, resp., induced by $p$ from the given orientation of $N$ at $y_{0}$. If $p \circ \gamma_{i j}$ reverses it, then the two framings correspond to opposite orientations of $V$, and the isotopy does not change this.

Next let us recall how the involution inv in (1.9) is defined (cf. [K6], p. 632). Given $\left[C, \bar{g}^{\#}, \tilde{g}\right] \in \pi_{m}\left(S^{n} \wedge(\Omega N)^{+}\right)$, inv retains the submanifold $C \subset S^{m}$ but changes its framing by $(-1)^{n} \cdot \alpha$ where the vector bundle automorphism $\alpha$ of the trivial bundle $C \times \mathbb{R}^{n}$ is determined by $T N$ and the homotopy $C \times I \rightarrow N$ which evaluates $\tilde{g}$ (cf. [K4], 3.1). In addition, we have to compose $\tilde{g}$ with the selfmap of $\Omega N$ which inverts the direction of loops.

Example 3.13 $(\mathbf{m}=\mathbf{n} \equiv \mathbf{0}(\mathbf{2}), N$ orientable). Here the framings (= coorientations) of the (isolated) points $x \in C$ remain unchanged, but we have to travel backwards along each loop $\tilde{g}(x)$.

Warning. In general the symmetry relation (1.9) does not necessarily extend to $\underline{\omega}^{\#}$ : the weaker invariant $\underline{\omega}^{\#}\left(f_{2}, f_{1}\right)$ may depend on more than just its weak counterpart $\underline{\omega}^{\#}\left(f_{1}, f_{2}\right)$.

A weaker version of our looseness obstruction $\omega^{\#}\left(f_{1}, f_{2}\right)$ is often much easier to handle and to compute. If we forget about embeddings of coincidence manifolds into $S^{m}$ and if we keep track only of stabilized normal bundles we obtain the invariants $\tilde{\omega}_{A}\left(f_{1}, f_{2}\right), A \in \pi_{1}(N)$, and

$$
\tilde{\omega}\left(f_{1}, f_{2}\right) \in \Omega_{m-n}^{\mathrm{fr}}(\Omega N) \cong \pi_{m+q}\left(S^{n+q} \wedge(\Omega N)^{+}\right), \quad q \gg 0,
$$

which lie in the $(m-n)$-dimensional framed bordism group of the loop space $\Omega N$ of $N$ (cf. [K4]). Similarly

$$
\omega\left(f_{1}, f_{2}\right):=\underline{\tilde{\omega}}\left(f_{1}, f_{2}\right), \quad \underline{\tilde{\omega}}_{A}\left(f_{1}, f_{2}\right) \quad \in \quad \Omega_{m-n}^{\mathrm{fr}}=\pi_{m-n}^{S}
$$


(compare [K2], 1.4) are the stabilized versions of $\underline{\omega}^{\#}\left(f_{1}, f_{2}\right)$ and $\underline{\omega}_{A}^{\#}\left(f_{1}, f_{2}\right)$.

In general this stabilization procedure leads to a loss of information; not so, however, in the dimension range $m<2 n-2$ where $\tilde{\omega}\left(f_{1}, f_{2}\right)$ is just as strong as $\omega^{\#}\left(f_{1}, f_{2}\right)$ and, in fact, is the complete looseness obstruction for the pair $\left(f_{1}, f_{2}\right)$ (cf. [K6], (16) and [K4], theorem 1.10).

\section{Selfcoincidences}

In this section we prove theorem 1.21 and further vanishing results and discuss their consequences.

Given any map $f:\left(S^{m}, x_{0}\right) \rightarrow\left(N, y_{0}\right)$ let us look at the coincidence data of the pair $(f, f)$. Clearly the map $\tilde{g}$ (cf. (3.4) ) is canonically nullhomotopic since $f_{1}=f_{2}=f$ in (3.5). For small generic approximations of $(f, f)$ the partial coincidence manifolds $C_{A}$ are empty (and hence nullbordant) whenever $A \in \pi_{1}(N)$ is nontrivial.

We conclude that

$$
\left.\omega^{\#}(f, f)=s_{*} \underline{\omega}_{0}^{\#}(f, f)\right)
$$

where

$$
s_{*}: \pi_{m}\left(S^{n}\right) \rightarrow \pi_{m}\left(S^{n} \wedge(\Omega N)^{+}\right)
$$

is induced by the inclusion of the constant loop into $\Omega N$ (recall also the notation of 3.12 .

Proof of theorem 1.21. We base our argument on the identity

$$
\underline{\omega}_{A}^{\#}(f, f)=\underline{\omega}_{A}^{\#}\left(f, y_{0}\right)+\underline{\omega}_{A}^{\#}\left(y_{0}, f\right)
$$

(valid for all $A \in \pi_{1}(N)$; cf. (1.13)) and on proposition 3.12, The assumptions in 1.21 just mean that the homomorphism $w_{1}: \pi_{1}(N) \rightarrow \mathbb{Z}_{2}$ (cf. 3.12) is not injective. In other words, there exists an element $A$ of $\pi_{1}(N)$ which is both nontrivial and orientation preserving. Then on the one hand $\underline{\omega}_{A}^{\#}(f, f)=0$. On the other hand 3.12 and (4.2) combine to show that $\underline{\omega}_{A}^{\#}(f, f)=\underline{\omega}_{0}^{\#}(f, f)$. Thus in view of (4.1) the full $\omega^{\#}$-invariant of the pair $(f, f)$ - and of every pair freely homotopic to it (cf. remark 1.32) - vanishes.

To complete the proof of theorem 1.21 note that

$$
\omega^{\#}(f, f)=\omega^{\#}\left(f, y_{0}\right)+\omega^{\#}\left(y_{0}, f\right)=(\mathrm{id}+\mathrm{inv})\left(\operatorname{deg}^{\#}(f)\right)
$$

(cf. (1.8), (1.9) and (1.10) ). Recall also corollary 1.16 .

Corollary 1.25 follows now from 1.3 , 1.4, (1.11), (1.13), 1.15 (to be discussed below), 1.21, 1.24 and the compatibility of $\underline{\omega}(f, f)$ with covering maps (compare (4.1)).

As for the conclusion of example 1.22 see also 3.13 . Similarly, the statement in example 1.23 is a consequence of theorem 1.21 and the following wellknown

Fact 4.3 (real Grassmann manifolds). Given integers $0<k<r$, the manifold $G_{r, k}$ of all $k$-dimensional linear subspaces of $\mathbb{R}^{r}$ enjoys the following properties: 
1. $\pi_{1}\left(G_{r, k}\right) \cong \mathbb{Z}_{2}$ if $r>2$;

2. $G_{r, k}$ is orientable if and only if $r$ is even;

3. $\operatorname{dim}\left(G_{r, k}\right)=k(r-k)$;

4. the Euler characteristic vanishes if $k \not \equiv r \equiv 0(2)$ and equals the binomial coefficient

in all other cases.

$$
\chi\left(G_{r, k}\right)=\left(\begin{array}{l}
{[r / 2]} \\
{[k / 2]}
\end{array}\right)>0
$$

Question 4.4. What about $\omega^{\#}(f, f)$ for maps into $G_{r, k}$ or $\tilde{G}_{r, k}$ when $r$ is odd and $k>2$ ?

Proof of theorem 1.15. A homotopy lifting argument shows that $(f, f)$ is loose by small deformation precisely if the pulled back vector bundle $f^{*}(T N)$ has a nowhere vanishing section over $S^{m}$ (cf. [DG] or [K7, 5.3) or, equivalently, if $f$ lifts to $S T(N)$ (cf. (1.14)). Thus (i) $\Longleftrightarrow$ (ii).

Recall also from [K7, 5.7 that $\pm E(\partial[f])$ equals the invariant $\underline{\omega}^{\#}(f, f)$ which is just as strong as $\omega^{\#}(f, f)$ (cf. (4.1)).

Next compare the fibre homotopy sequence of $S T(N)$ and of the configuration space $\tilde{C}_{2}(N)=N \times N \backslash \Delta$ (cf. [K7, 5.4). We see that (iii) $\Longrightarrow$ (i) provided the induced homomorphism $j_{*}$ (cf. [1.24) is injective. This is the case e.g. when $N=\mathbb{R} \mathbb{P}(n)$.

Finally recall that (iv) implies (iii) when $N=S^{n}$; this is a special case of [6], 1.12 .

Next we prove corollary 1.16, Clearly $E$ (cf. (1.14)) is injective if $m=n$ or $m \leq 2 n-3$ or $n=2\left(\right.$ since $\pi_{m-1}\left(S^{1}\right)=0$ for $\left.m>2\right)$. Moreover $\partial\left(\pi_{m}(N)\right)=0$ if $n$ is odd since then the fibration $S T(N) \rightarrow N$ allows a section over each compactum (compare (1.14)). Thus our corollary follows from

Proposition 4.5. Consider the suspension homomorphism

$$
E: \pi_{m-1}\left(S^{n-1}\right) \rightarrow \pi_{m}\left(S^{n}\right)
$$

and

$$
E^{\infty}: \pi_{m-1}\left(S^{n-1}\right) \rightarrow \pi_{m-n}^{S}
$$

and assume that $n$ is even. If $m \leq n+3$ or if $m=n+4 \neq 8,10$ then $E$ and $E^{\infty}$ are injective. If $m=8$ and $n=4$, then $E$ is injective, but $E^{\infty}$ is not.

Proof. We will use Toda's tables [T. Since $E^{\infty}: \pi_{5}\left(S^{3}\right) \cong \mathbb{Z}_{2} \rightarrow \pi_{2}^{S} \cong \mathbb{Z}_{2}$ is onto and hence bijective, it remains only to study the (nonstable) cases where $n=4$ and $m=7$ or 8 . The groups in the exact EHP-sequence

$$
\pi_{7}\left(S^{3}\right) \stackrel{E}{\longrightarrow} \pi_{8}\left(S^{4}\right) \stackrel{H}{\longrightarrow} \pi_{8}\left(S^{7}\right) \stackrel{P}{\longrightarrow} \pi_{6}\left(S^{3}\right) \stackrel{E}{\longrightarrow} \pi_{7}\left(S^{4}\right) \longrightarrow \ldots
$$

(cf. [W], XII, 2.3) have order 2,4,2 and 12, resp.; hence both homomorphisms $E$ are injective here. Moreover the kernel of

$$
E^{\infty}: \pi_{7}\left(S^{4}\right) \cong \mathbb{Z} \oplus \mathbb{Z}_{12} \rightarrow \pi_{3}^{S} \cong \mathbb{Z}_{24}
$$


is generated by $\left[\iota_{4}, \iota_{4}\right]$ and has a trivial intersection with $E\left(\pi_{6}\left(S^{3}\right)\right)=\{0\} \oplus$ $\mathbb{Z}_{12}$. Our claim follows for $(m, n)=(7,4)$ and similarly for $(m, n)=(8,4)$ (since $\left.\pi_{7}\left(S^{3}\right) \neq 0=\pi_{4}^{S}\right)$.

Corollary 4.6. There exists a map $f: S^{8} \rightarrow \mathbb{R P}(4)$ such that $\omega^{\#}(f, f) \neq 0$ but $\tilde{\omega}(f, f)=0$. Of course the corresponding liftings $\tilde{f}: S^{8} \rightarrow S^{4}$ have the same property.

Proof. The groups in the exact sequence

$$
\pi_{8}\left(S T\left(S^{4}\right)\right) \rightarrow \pi_{8}\left(S^{4}\right) \stackrel{\partial}{\longrightarrow} \pi_{7}\left(S^{3}\right)
$$

(cf. (1.14) ) have order 2 (cf. $[\mathrm{P}$ ), 4 and 2, resp.; thus there is an element $[\tilde{f}] \in$ $\pi_{8}\left(S^{4}\right)$ such that $\partial([\tilde{f}])$ and hence $\underline{\omega} \#(\tilde{f}, \tilde{f})=E(\partial[\tilde{f}])$ is nontrivial, but $\omega(\tilde{f}, \tilde{f})=$ $\underline{\tilde{\omega}}(\tilde{f}, \tilde{f}) \in \pi_{4}^{S}=0$ (compare also (4.10) $)$.

Next we want to explore the fact that it is often easier to compute the stabilized versions of our $\omega$-invariants. Indeed they just sum up the contributions of the partial coincidence manifolds $C_{A}$ (not registering their linkings); also we just multiply a given bordism class by -1 if we compose the framing with a reflection of $\mathbb{R}^{n}$.

Proposition 4.7. Given $[f] \in \pi_{m}(N)$, the stabilized invariant $\tilde{\omega}(f, f)$ is determined by $\underline{\tilde{\omega}}(f, f)$, i.e. by

$$
\omega(f, f)=\chi(N) \omega\left(f, y_{0}\right)=\omega\left(f, y_{0}\right)+E^{\infty}\left(\operatorname{coll}_{*}([f])\right)= \pm E^{\infty} \circ \partial([f]) .
$$

(Here $\chi(N)$ denotes the Euler characteristic of $N$; for coll $_{*}$ see 3.10.) If the stable suspension homomorphism $E^{\infty}: \pi_{m-1}\left(S^{n-1}\right) \rightarrow \pi_{m-n}^{S}$ is injective (or if $m \leq n+3)$ then the conditions (i)-(v) in theorem 1.15 are all equivalent to

(vi) $\omega(f, f)=0$.

Proof. The first identity was already established in [K2, 2.2; the corresponding claim for $\omega^{\#}(f, f)$ (see [K6], 5.1) is more complicated and not so easy to use in calculations.

The second identity follows from (1.13) and 3.10, Furthermore note that $\omega(f, f)=\underline{\tilde{\omega}}(f, f)$ is the stable suspension of $\underline{\omega}^{\#}(f, f)= \pm E(\partial([f]))$ (cf. [K7], 5.7).

If $m \leq n+3$ and $n$ is even, then $E^{\infty}$ is injective (cf. 4.5). If $n$ is odd, all conditions (i)-(vi) hold automatically.

Proposition 4.8. Assume that $k:=\# \pi_{1}(N) \geq 2$. Consider arbitrary maps $f, f_{1}, f_{2}:\left(S^{m}, x_{0}\right) \rightarrow\left(N, y_{0}\right)$.

If $N$ is orientable, then

$$
\chi(N) \cdot \omega\left(f_{1}, f_{2}\right) \quad=0 \quad(\text { cf. (3.14) })
$$

and in particular

$$
\left.\chi(N) \cdot E^{\infty}\left(\operatorname{coll}_{*}([f])\right)=0 \quad \text { (cf. 3.10) }\right) .
$$

If $N$ is not orientable then $E^{\infty}\left(\operatorname{coll}_{*}([f])\right)=0$ and

$$
(\chi(N)-1) \omega\left(f_{1}, f_{2}\right)=0 ;
$$


if in addition $k>2$, then $\omega\left(f_{1}, f_{2}\right)=0$.

Proof. Note that $\omega\left(y_{0}, f\right)=E^{\infty}\left(\operatorname{coll}_{*}([f])\right)($ cf. 3.10). If $N$ is orientable, then

$$
0=\omega(f, f)=\omega\left(f, y_{0}\right)+\omega\left(y_{0}, f\right)=\chi(N) \cdot \omega\left(f, y_{0}\right)
$$

(cf. 1.21] (1.13) and 4.7). Thus multiplication by the Euler characteristic annihilates also $\omega\left(y_{0}, f\right)=-\omega\left(f, y_{0}\right)$ and hence $\omega\left(f_{1}, f_{2}\right)$ (by (1.13)).

If $N$ is not orientable, then it follows from proposition 3.12 that

$$
\underline{\tilde{\omega}}_{A}\left(f, y_{0}\right)=\underline{\tilde{\omega}}_{0}\left(f, y_{0}\right) \text { and } \underline{\tilde{\omega}}_{A}\left(y_{0}, f\right)=\varepsilon_{A} \cdot \underline{\tilde{\omega}}_{0}\left(y_{0}, f\right)
$$

for every $A \in \pi_{1}(N)$ where $\varepsilon_{A}=+1$ or -1 according as $A$ is orientation preserving or reversing. Thus

$$
\omega\left(y_{0}, f\right)=\sum_{A} \underline{\tilde{\omega}}_{A}\left(y_{0}, f\right)=0 .
$$

Moreover

$$
\underline{\tilde{\omega}}_{A}(f, f)=\underline{\tilde{\omega}}_{A}\left(f, y_{0}\right)+\underline{\tilde{\omega}}_{A}\left(y_{0}, f\right)=0
$$

whenever $A \neq 0$ (cf. (1.13) and (4.1)). If in addition $k>2$ then there exist both orientation preserving and reversing $A \neq 0$ so that $\underline{\tilde{\omega}}_{0}\left(f, y_{0}\right)=-\varepsilon_{A} \cdot \underline{\tilde{\omega}}_{0}\left(y_{0}, f\right)$ both for $\varepsilon_{A}=+1$ and $\varepsilon_{A}=-1$; hence $\omega\left(f, y_{0}\right)$, being an even multiple of an element of order 2 , vanishes, and so does $\omega\left(f_{1}, f_{2}\right)$, again by (1.13). If $\pi_{1}(N)$ consists only of 0 and $A \neq 0$, then

$$
\underline{\tilde{\omega}}_{0}\left(f, y_{0}\right)=\underline{\tilde{\omega}}_{A}\left(f, y_{0}\right)=-\underline{\tilde{\omega}}_{A}\left(y_{0}, f\right)=\underline{\tilde{\omega}}_{0}\left(y_{0}, f\right)
$$

and

$$
\omega\left(f, y_{0}\right)=2 \underline{\tilde{\omega}}_{0}\left(f, y_{0}\right)=\omega(f, f)=\chi(N) \omega\left(f, y_{0}\right)
$$

(cf. 4.7), so that multiplication by $\chi(N)-1$ annihilates $\omega\left(f, y_{0}\right)$ and hence also $\omega\left(f_{1}, f_{2}\right)=\omega\left(f_{1}, y_{0}\right)($ cf. (4.10) $)$.

Given a map $f:\left(S^{m}, x_{0}\right) \rightarrow\left(N, y_{0}\right)$, consider a lifting $\tilde{f}:\left(S^{m}, x_{0}\right) \rightarrow\left(\tilde{N}, \tilde{y}_{0}\right)$ to the universal covering space $p: \tilde{N} \rightarrow N$.

Lemma 4.13. We have

$$
\underline{\tilde{\omega}}_{0}\left(f, y_{0}\right)=\omega\left(\tilde{f}, \tilde{y}_{0}\right) \quad \text { and } \quad \underline{\tilde{\omega}}_{0}\left(y_{0}, f\right)=\omega\left(\tilde{y}_{0}, \tilde{f}\right) \text {. }
$$

Proof. In the Nielsen decomposition

$$
f^{-1}\left(\left\{y_{0}\right\}\right)=\tilde{f}^{-1}\left(p^{-1}\left(\left\{y_{0}\right\}\right)\right)=\tilde{f}^{-1}\left(\left\{A \tilde{y}_{0} \mid A \in \pi_{1}(N)\right\}\right)
$$

of the relevant coincidence manifold, $\tilde{f}^{-1}\left(\left\{\tilde{y}_{0}\right\}\right)$ is the component indexed by $A=$ 0 .

Let us apply this to the special case $\tilde{N}=S^{n}$ and $N=\mathbb{R} \mathbb{P}(n), n$ even. Then

$$
\omega(f, f)=2 \underline{\tilde{\omega}}_{0}\left(y_{0}, f\right)=2 \omega\left(\tilde{y}_{0}, \tilde{f}\right)=2 E^{\infty}([\tilde{f}])
$$

(cf. (4.12), 4.13, 3.10 and (3.15)). This establishes the claim which follows theorem 1.31. Also if $m=2 n-1$ and e.g. $n=4,8,12,14,16$ or 20 , then $\operatorname{ker} E^{\infty} \cong \mathbb{Z}$ 
and $E^{\infty}$ maps $\pi_{m}\left(S^{n}\right)$ onto the stable stem $\pi_{n-1}^{S}$ which contains elements of order greater than 2 (cf. [T]). This proves the statement in example 1.26.

Similarly, (4.12) shows also (together with theorem 1.21) that $\omega(f, f) \in$ $2 \cdot \pi_{m-n}^{S}$ whenever $N$ is not simply connected. This (together with 4.8) establishes (1.27).

Finally let us discuss example [1.28. According to fact 4.3. the Grassmann manifold $G_{5,2}$ is not orientable, 6-dimensional and its Euler characteristic equals 2. Our claim follows from proposition 4.8 and the Freudenthal suspension theorem.

\section{Nielsen numbers}

Definition 5.1. Given $f_{1}, f_{2}: S^{m} \rightarrow N$, the "strong" Nielsen number $\mathrm{N}^{\#}\left(f_{1}, f_{2}\right)$ (and its stabilized analogue $\mathrm{N}\left(f_{1}, f_{2}\right)$, resp.) is the number of elements $A \in \pi_{1}(N)$ such that $\omega_{A}^{\#}\left(f_{1}, f_{2}\right)$ (and $\tilde{\omega}_{A}\left(f_{1}, f_{2}\right)$, resp.) does not vanish (cf. (3.8) and (3.14) ).

Since generic coincidence manifolds are compact these Nielsen numbers are always finite.

Proof of theorem 1.30. If $\omega^{\#}\left(f_{1}, f_{2}\right)$ (or $\tilde{\omega}\left(f_{1}, f_{2}\right)$, resp.) vanishes, then so do all the partial invariants $\omega_{A}^{\#}\left(f_{1}, f_{2}\right)$ (or $\tilde{\omega}_{A}\left(f_{1}, f_{2}\right)$, resp.), $A \in \pi_{1}(N)$, as well as the corresponding Nielsen number. The converse is also obvious in the stabilized setting. However, $\omega^{\#}\left(f_{1}, f_{2}\right)$ keeps track also of embeddings and of possible linking phenomena among the partial coincidence submanifolds $\mathrm{C}_{A}\left(f_{1}, f_{2}\right)$ in $S^{m}$.

Assume that $\mathrm{N}^{\#}\left(f_{1}, f_{2}\right)=0$. Then all triples $\left(\mathrm{C}_{A}\left(f_{1}, f_{2}\right), \bar{g}_{A}^{\#}, \tilde{g}_{A}\right)($ cf. (3.8) $)$ admit individual nullbordisms in $S^{m} \times I$. Conceivably these can not be fitted together disjointly to yield the full embedded nullbordism required to show that $\omega^{\#}\left(f_{1}, f_{2}\right)$ vanishes. In fact, it is an open question whether the first claim in 1.30 still holds when the common domain of $f_{1}$ and $f_{2}$ is not a sphere.

But here we can use the additive structure of homotopy groups. As in (1.8) we have for all $A \in \pi_{1}(N)$

$$
0=\omega_{A}^{\#}\left(f_{1}, f_{2}\right)=\omega_{A}^{\#}\left(f_{1}, f_{1}\right)+\omega_{A}^{\#}\left(y_{0}, f_{2}-f_{1}\right)
$$

The claim of our theorem is obvious when $\pi_{1}(N)=0$ or in the selfcoincidence case since only $A=0$ plays a rôle there (cf. (4.1) $)$. If $A \neq 0$ then $\omega_{A}^{\#}\left(f_{1}, f_{1}\right)=0$ and hence $\omega_{A}^{\#}\left(y_{0}, f_{2}-f_{1}\right)=0(\mathrm{cf}$. (5.2) $)$. This implies - in this special root case - that the full invariant $\omega^{\#}\left(y_{0}, f_{2}-f_{1}\right)$ vanishes; indeed, a nullbordism of $\mathrm{C}_{A}\left(y_{0}, f_{2}-f_{1}\right)$ gives rise to disjoint "parallel" nullbordisms of all the other partial coincidence manifolds $\mathrm{C}_{A^{\prime}}\left(y_{0}, f_{2}-f_{1}\right), A^{\prime} \in \pi_{1}(N)$ (compare the proof of 3.12 or also [K6], 4.3). Thus in turn $\omega^{\#}\left(f_{1}, f_{2}\right)=\omega^{\#}\left(f_{1}, f_{1}\right)$ (cf. (1.8) $)$ and we are back in the selfcoincidence case.

Proof of theorem 1.31. If $k=\# \pi_{1}(N)$ is infinite, then all pairs $\left(f_{1}, f_{2}\right)$ are loose and hence all Nielsen numbers vanish (cf. (3.1)). If $k>2$ or if $k=2$ and $N$ is orientable, then always $\omega^{\#}\left(f_{1}, f_{1}\right)=0$ (cf. 1.21); therefore $\omega^{\#}\left(f_{1}, f_{2}\right)=$ $\omega^{\#}\left(y_{0}, f_{2}-f_{1}\right)(\mathrm{cf} .(5.2))$ and $\tilde{\omega}\left(f_{1}, f_{2}\right)=\tilde{\omega}\left(y_{0}, f_{2}-f_{1}\right)$. Again 3.12 implies that 
the corresponding Nielsen numbers can assume only the values 0 and $k$ in this root case. For the remaining cases compare corollary 1.25.

Remark 5.3. Consider the split exact sequence

$$
0 \rightarrow \operatorname{ker}(\text { forg }) \hookrightarrow \pi_{m}\left(S^{n} \wedge(\Omega N)^{+}\right) \stackrel{\text { forg }}{\longrightarrow} \bigoplus_{A \in \pi_{1}(N)} \pi_{m}\left(S^{n} \wedge A^{+}\right) \rightarrow 0 ;
$$

here, given any element $\omega^{\#}=\left[C, \bar{g}^{\#}, \tilde{g}\right] \in \pi_{m}\left(S^{n} \wedge(\Omega N)^{+}\right)$, forg $\left(\omega^{\#}\right)$ keeps track only of the individual $A$-components $\left[C_{A}=\tilde{g}^{-1}(A), \bar{g}^{\#}|, \tilde{g}|\right]$ and forgets about possible linkings. As we will see below, the kernel of forg can be highly nontrivial if $\# \pi_{1}(N)>1$.

Consider also the function $\mathrm{N}^{\#}$ which counts the nontrivial $A$-components. Clearly it vanishes on ker(forg) and, in addition, can often assume all integer values between 0 and $\# \pi_{1}(N)$. This shows that theorems 1.30 and 1.31 impose strong restrictions on those $\omega^{\#}$-values which can actually be realized by pairs $\left(f_{1}, f_{2}\right)$ of maps.

Example 5.4 (real projective spaces). Consider $N=\mathbb{R} \mathbb{P}(n)$ and its double cover $\tilde{N}=S^{n}$. There is a wellknown isomorphism

$$
\pi_{m}\left(S^{n} \wedge(\Omega N)^{+}\right) \cong \pi_{m}\left(S_{1}^{n} \vee S_{2}^{n} \vee \tilde{N}, \tilde{N}\right)
$$

(cf. e.g. [K6], (61)), and the forgetful map forg (cf. [5.3) corresponds to the homomorphism

$$
\pi_{m}\left(\bigvee^{2} S^{n} \vee \tilde{N}\right) \rightarrow \bigoplus_{i=1}^{2} \pi_{m}\left(S_{i}^{n} \vee \tilde{N}\right)
$$

which is induced by the obvious projections. Let $\iota_{1}, \iota_{2}$ and $\iota_{0}$ be represented by the inclusions of the three spheres into their wedge. Then the summands of

$$
\operatorname{ker}(\text { forg }) \cong \pi_{m}\left(S^{2 n-1}\right) \oplus\left(\pi_{m}\left(S^{3 n-2}\right)\right)^{4} \oplus \ldots
$$

in the Hilton decomposition of $\pi_{m}\left(S_{1}^{n} \vee S_{2}^{n} \vee \tilde{N}\right)$ (cf. [W], XI, 6) corresponds precisely to those basic products which involve both $\iota_{1}$ and $\iota_{2}$.

\section{References}

[B] R. Brown, Wecken properties for manifolds. Contemp. Math. 152 (1993), 9-21.

[BS] R. Brown and H. Schirmer, Nielsen coincidence theory and coincidence-producing maps for manifolds with boundary. Topology Appl. 46 (1992), 65-79.

[D] A. Dold, The fixed point index of fibre-preserving maps. Inv. Math. 25 (1974), 281-297.

[DG] A. Dold and D.L. Gonçalves, Self-coincidence of fibre maps. Osaka J. Math. 42 (2005), 291-307.

[GR1] D.L. Gonçalves and D. Randall, Self-coincidence of maps from $S^{q}$-bundles over $S^{n}$ to $S^{n}$. Bol. Soc. Mat. Mexicana (3) 10 (2004), 181-192. 
[GR2] D.L. Gonçalves and D. Randall, Self-coincidence of mappings between spheres and the strong Kervaire invariant one problem. Comptes Rendus Math. Acad. Sci. Paris, Ser. I 342 (2006), 511-513.

[GW] D.L. Gonçalves and P. Wong, Wecken property for roots. Proc. AMS (9) 133 (2005), 2779-2782.

[GH] M. Greenberg and J. Harper, Algebraic topology - a first course. Math. Lect. Notes Series, Addison-Wesley Publ. Co., 1981.

[K1] U. Koschorke, Vector fields and other vector bundle monomorphisms - a singularity approach. Lect. Notes in Math. 847 (1981), Springer-Verlag.

[K2] U. Koschorke, Selfcoincidences in higher codimensions. J. reine angew. Math. 576 (2004), 1-10.

[K3] U. Koschorke, Linking and coincidence invariants. Fund. Math. 184 (2004), 187203.

[K4] U. Koschorke, Nielsen coincidence theory in arbitrary codimensions. J. reine angew. Math. 598 (2006), 211-236.

[K5] U. Koschorke, Geometric and homotopy theoretic methods in Nielsen coincidence theory. Fixed Point Theory and Appl. (2006), Article ID 84093, 15 pp.

[K6] U. Koschorke, Nonstabilized Nielsen coincidence invariants and Hopf-Ganea homomorphisms. Geom. Top. 10 (2006), 619-665.

[K7] U. Koschorke, Minimizing coincidence numbers of maps into projective spaces. Memorial Volume in honor of H. Zieschang, Geom. Top., to appear.

[P] G.F. Paechter, The groups $\pi_{r}\left(V_{n, m}\right)$ (I). Quarterly J. Math. Oxford (2) 7 (1956), 249-268.

[T] H. Toda, Composition methods in homotopy groups of spheres. Princeton Univ. Press, 1962.

[W] G. Whitehead, Elements of homotopy theory. Graduate Texts in Mathematics (1978), Springer-Verlag.

[Wy] A. Wyler, Sur certaines singularités d'applications de variétés topologiques. Comm. Math. Helv. 42 (1967), 28-48.

Ulrich Koschorke

Fachbereich Mathematik

Universität Siegen

57068 Siegen - Germany

e-mail: koschorke@mathematik.uni-siegen.de 